\title{
ROBUST SECURE PRECODING AND ANTENNA SELECTION: A PROBABILISTIC OPTIMIZATION APPROACH FOR INTERFERENCE EXPLOITATION
}

\author{
Zhongxiang Wei and Christos Masouros
}

\author{
Department of Electronic and Electrical Engineering \\ University College London, London
}

\begin{abstract}
In this paper, to realize a power-efficient, user-centric and physical layer security-addressing system, we investigate total power minimization by jointly designing antenna selection and secure precoding for distributed antenna (DA) systems. Different from the conventional artificial noise (AN)-aided secure transmission, where AN is treated as an undesired element for the intended receiver (IR), we design AN such that it is constructive to the IR while keeping destructive to the eavesdroppers (Eves). Importantly, we investigate a practical scenario, where the IR and Eves' channel state information (CSI) is imperfectly obtained. To handle the CSI uncertainties, we solve the problem in a probabilistic manner, which statistically satisfies the IR' signal-to-interference-and-ratio (SINR) requirement by use of constructive AN and addresses security against the Eves. Simulation demonstrates our algorithm consumes much less power compared to the centralized antenna (CA) systems, as well as the DA systems with conventional AN processing. Last but not least, a user-centric and on-demand structure is presented by the algorithm, thanks to the adaptive DAs activation/deactivation mechanism.
\end{abstract}

Index Terms - Distributed antenna, Antenna selection, Secure precoding, Robust optimization, Constructive interference

\section{INTRODUCTION}

Centralized multiple-input multiple-output (MIMO) has been considered as a key technology to provide high throughput [1] and additional spatial diversity for enhancing physical layer (PHY) security [2]. However, it incurs high power consumption and suffers from an equal level of path loss (PL) from the antenna array to one user [3]. To this end, distributed antenna (DA) systems have been considered as an alternative [4] to realize a user-centric and power efficient network. By geographically positioning the antennas and hence shortening the communication distance, DA systems can reduce the PL and facilitate an on-demand network by only activating those DAs contributing the most. The concepts of user-centric DA are

This work was supported by the Engineering and Physical Sciences Research Council, UK, under project EP/R007934/1. also suited for communications in industry, where antennas can be distributed in the ceilings of large factories to effectively extend network coverage, and is therefore a key contender for industry Internet of Things [5].

As communication distance is shortened in DA systems, however, it is easier for eavesdroppers (Eves) to seize the confidential signal transmitted to the intended receiver (IR), and PHY security becomes more challenging [6]. In the past decades, PHY security has been extensively investigated to secure wireless communications, where artificial noise (AN) is generated to interfere potential Eves in isotropic [7] or spatial manner [8]. However, it is worth noting that AN is always considered as a harmful element at the IR and its leakage needs to be minimized [9]. If $\mathrm{AN}$ can be beneficial to the IR based on the concept of constructive interference (CI), it helps improve the IR's signal-to-interference-plus-noise ratio (SINR). Symbol-level CI was introduced by [10], and the authors in [11] further proposed a scheme to utilize AN in a centralized antenna (CA) system. However, the fully activated antennas in [11] lead to enormous power consumption. Motivated by the aforementioned issues, in this paper, we present joint design of DA selection and secure precoding to minimize total power consumption, subjected to PHY security constraints. Importantly, we consider a practical scenario and solve it by probabilistic robust optimizations. Our contributions are summarized as follows:

a. We investigate power minimization problem under the IR's quality-of-service (QoS) requirement and PHY security constraints against Eves. DA selection and precoding are jointly designed to fully utilize the additional degrees of freedom in DAs' activation/deactivation and beneficial effect of $\mathrm{AN}$, which is shown to significantly reduce the total power.

b. We exploit joint DA selection and robust precoding in a practical scenario, where the IR and Eves' channel state information (CSI) is imperfectly obtained. Explicitly, the CSI uncertainties are processed in a probabilistic manner: the IR's QoS requirement and the security against the Eves are addressed by chance constrained formulations. A lowcomplexity algorithm is proposed to minimize total power consumption, and at the same time $\mathrm{AN}$ is kept constructive to the IR whereas destructive to the Eves.

c. A user-centric network structure is demonstrated by 
the proposed scheme, compared to the CA counterpart. Explicitly, the working status of each DA is flexibly determined by the IR and Eves' positions to provide on-demand services, thanks to the DA selection mechanism.

Notations: $\boldsymbol{A}^{H}, \boldsymbol{A}^{T}$ and $\operatorname{Tr}(\boldsymbol{A})$ denote the Hermitian transpose, transpose and trace of matrix $\boldsymbol{A}$. diag $(\cdot)$ returns a diagonal matrix. $\boldsymbol{A} \succeq 0$ means $\boldsymbol{A}$ is a positive semi-definite matrix. $\|\cdot\|_{p}$ means the p-norm. $\boldsymbol{I}_{n}$ means a $n$-by- $n$ identity matrix. Subscript $\mathrm{R}$ and I represent the real part and imaginary parts. $\mathbb{C}^{N \times M}$ and $\mathbb{H}^{N \times M}$ denote sets of all $N \times M$ matrices and Hermitian matrices with complex entries.

\section{SYSTEM MODEL AND CONSTRUCTIVE INTERFERENCE}

$N$ DAs are connected to the central unit through a noise-free wired front-haul for cooperative communications. Confidential message is transmitted to the IR in the presence of $K$ Eves. The IR and Eves are all equipped with single antenna for simplicity [12]. CSI is obtained by channel estimation in the training phase [4]. Without loss of generality, we assume that all the DAs share the same drain efficiency [13].

We now briefly introduce the concept of CI. CI pushes the received signals aways from the detection threshold [10]. The increased distance to the detection threshold can effectively improve the receiving performance. Denote $\boldsymbol{w} \in \mathbb{C}^{N \times 1}$ and $\boldsymbol{z} \in \mathbb{C}^{N \times 1}$ as precoding and $\mathrm{AN}$ at the transmitter side. Denote the real channels as $\boldsymbol{h}_{d}=\hat{\boldsymbol{h}_{d}}+\boldsymbol{e}_{d}$ and $\boldsymbol{h}_{k}=\hat{\boldsymbol{h}_{k}}+\boldsymbol{e}_{k}, \forall k \in K$, where $\hat{\boldsymbol{h}_{d}} \in \mathbb{C}^{N \times 1}$ denotes the estimated channel between the DAs and the IR with estimation error $\boldsymbol{e}_{d} \in \mathbb{C}^{N \times 1}$, and $\hat{\boldsymbol{h}_{k}} \in \mathbb{C}^{N \times 1}$ denotes the estimated channel between the DAs and the $k$-th Eve with error $e_{k} \in \mathbb{C}^{N \times 1}$. Assume the channel estimation error of the IR and the $k$-th Eve follows normal distribution $\boldsymbol{e}_{d} \sim \mathcal{C} \mathcal{N}\left\{0, \sigma_{d}^{2}\right\}$ and $\boldsymbol{e}_{k} \sim \mathcal{C N}\left\{0, \sigma_{k}^{2}\right\}, \forall k \in K$ [14]. Denote $x_{d}=d e^{j \phi_{d}}$ as the information-bearing symbol transmitted for the IR. The received signal at the IR and the $k$-th Eve can be calculated as

$$
y_{d}=\boldsymbol{h}_{d}^{T}\left(\boldsymbol{w} x_{d}+\boldsymbol{z}\right)+n_{d}, y_{k}=\boldsymbol{h}_{k}^{T}\left(\boldsymbol{w} x_{d}+\boldsymbol{z}\right)+n_{k},
$$

where $n_{d} \sim \mathcal{C N}\left(0, \sigma_{n}^{2}\right)$ and $n_{k} \sim \mathcal{C N}\left(0, \sigma_{n}^{2}\right)$ denote the Additive white Gaussian noises (AWGN) at the IR and the $k$-th Eve, respectively. Conventionally, the received SINR at the IR and $k$-th Eve are denoted as $\Gamma_{d}=\frac{\left|\boldsymbol{h}_{d}^{T} \boldsymbol{w}\right|^{2}}{\sigma_{n}^{2}+\left|\boldsymbol{h}_{d}^{T} \boldsymbol{z}\right|^{2}}, \Gamma_{k}=$ $\frac{\left|\boldsymbol{h}_{k}^{T} \boldsymbol{w}\right|^{2}}{\sigma_{n}^{2}+\left|\boldsymbol{h}_{k}^{T} \boldsymbol{z}\right|^{2}}$, where the AN is treated as an undesired element at the IR. By contrast, the principle of constructive AN is to rotate the phase of the AN at transmitter and to align it with the desired signal at the IR [15]. Since the transmitted signal can be also written as $\left(\boldsymbol{w}+\boldsymbol{z} e^{-j \phi_{d}}\right) x_{d}$, according to the geometric interpretation in Fig. 1, the requirement of generating constructive AN can be given as

$$
\begin{aligned}
\left|\Im\left\{\boldsymbol{h}_{d}^{T} \boldsymbol{w}+\boldsymbol{h}_{d}^{T} \boldsymbol{z} e^{-j \phi_{d}}\right\}\right| \leq \\
\quad\left(\Re\left\{\boldsymbol{h}_{d}^{T} \boldsymbol{w}+\boldsymbol{h}_{d}^{T} \boldsymbol{z} e^{-j \phi_{d}}\right\}-\sigma \sqrt{\Gamma_{d}}\right) \tan \theta,
\end{aligned}
$$

where $\theta=\pi / M$ with constellation size $M$ [16]. It can be seen from (2) that the AN becomes a beneficial element to the IR and the IR' SINR $\Gamma_{d}$ is also embedded. Since the AN contributes to the useful signal power, the received SINR of the IR becomes into

$$
\gamma_{d}=\frac{\left|\boldsymbol{h}_{d}^{T}\left(\boldsymbol{w}+\boldsymbol{z} e^{-j \phi_{d}}\right)\right|^{2}}{\sigma_{n}^{2}},
$$

where the transmission power can be efficiently reduced to achieve a target SINR requirement.

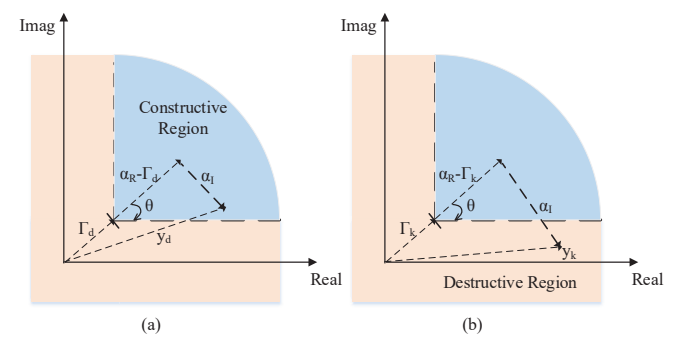

Fig. 1. Constructive and destructive AN for the IR and Eves with QPSK modulation.

\section{POWER EFFICIENT DA SELECTION AND SECURE PRECODING WITH IMPERFECT CSI}

Problem formulation is given in 3.1 and its solution is presented in 3.2 .

\subsection{Problem Formulation}

Define $w_{n}$ as the $n$-th element of the precoding vector $\boldsymbol{w}$ and $z_{n}$ as the $n$-th element of the AN $z$. Define DA selection vector $\boldsymbol{t}$, whose element $t_{n}=\{0,1\}$ means the $n$-th DA is deactivated or activated, respectively. Let $p_{\text {on }}$ and $p_{\text {off }}$ represent the power consumption of each activated/deactivated $\mathrm{DA}$, and $p_{D A}$ represent the maximum available transmission power at each DA. Let $\overline{\Gamma_{d}}$ and $\overline{\Gamma_{k}}$ denote the SINR requirement for the IR and physical layer security against the $k$-th Eve. Accordingly, the problem is formulated as

$$
\begin{aligned}
& P 1: \underset{\boldsymbol{w}, \boldsymbol{z}, \boldsymbol{t}}{\operatorname{argmin}} \frac{\left|\boldsymbol{w}+\boldsymbol{z} e^{-j \phi_{d}}\right|^{2}}{\alpha}+\sum_{n=1}^{N}\left(t_{n} p_{o n}+\left(1-t_{n}\right) p_{o f f}\right) \\
& \text { s.t }(C 1):\left|w_{n}+z_{n} e^{-j \phi_{d}}\right|^{2} \leq t_{n} p_{D A}, \forall n \in N, \\
& \quad(C 2): t_{n}=\{0,1\}, \forall n \in N, \\
& \quad(C 3): \operatorname{Pr}\left\{\Gamma_{d} \geq \overline{\Gamma_{d}} \mid \boldsymbol{e}_{d}\right\} \geq \eta_{d}, \forall k \in K, \\
& \quad(C 4): \operatorname{Pr}\left\{\Gamma_{k} \leq \overline{\Gamma_{k}} \mid \boldsymbol{e}_{k}\right\} \geq \eta_{k}, \forall k \in K,
\end{aligned}
$$


where $\alpha$ is the drain efficiency of the DAs. (C2) constrains the selection vector to binary (on/off) elements. (C3) guarantees that the SINR at the IR greater than the preset threshold $\overline{\Gamma_{d}}$ with probability $\eta_{d}$, while $(C 4)$ guarantees the $k$-th Eve's SINR lower than the threshold $\overline{\Gamma_{k}}$ with probability $\eta_{k}, \forall k \in$ $K$.

\subsection{Solution to the Problem}

We first need to handle the probabilistic constraints $(C 3)$ and $(C 4)$. Define $\boldsymbol{u}=\boldsymbol{w}+\boldsymbol{z} e^{-j \phi_{d}}$. Under the provision of CI, constraint $(C 3)$ can be written as two inequities

$$
\left\{\begin{array}{l}
\operatorname{Pr}\left\{\boldsymbol{a}_{\boldsymbol{d}, \mathbf{1}}^{T}\left[\boldsymbol{u}_{R} ; \boldsymbol{u}_{I}\right] \leq-\sigma_{n} \sqrt{\overline{\Gamma_{d}}} \tan \theta \mid \boldsymbol{e}_{d}\right\} \geq \eta_{d} \\
\operatorname{Pr}\left\{\boldsymbol{a}_{\boldsymbol{d}, \mathbf{2}}^{T}\left[\boldsymbol{u}_{R} ; \boldsymbol{u}_{I}\right] \leq-\sigma_{n} \sqrt{\overline{\Gamma_{d}}} \tan \theta \mid \boldsymbol{e}_{d}\right\} \geq \eta_{d}
\end{array}\right.
$$

where $\boldsymbol{a}_{d, 1}=\left[\hat{\boldsymbol{h}_{I, d}}-\hat{\boldsymbol{h}_{R, d}} \tan \theta+\boldsymbol{e}_{I, d}-\boldsymbol{e}_{R, d} \tan \theta ; \hat{\boldsymbol{h}_{R, d}}+\right.$ $\left.\hat{\boldsymbol{h}_{I, d}} \tan \theta+\boldsymbol{e}_{R, d}+\boldsymbol{e}_{I, d} \tan \theta\right]$, and $\boldsymbol{a}_{d, 2}=\left[-\hat{\boldsymbol{h}_{I, d}}-\boldsymbol{e}_{I, d}-\right.$ $\left.\hat{\boldsymbol{h}_{R, d}} \tan \theta-\boldsymbol{e}_{R, d} \tan \theta ;-\hat{\boldsymbol{h}_{R, d}}-\boldsymbol{e}_{R, d}+\hat{\boldsymbol{h}_{I, d}} \tan \theta+\boldsymbol{e}_{I, d} \tan \theta\right]$. It is easy to obtain that the $N$-dimensional normal distributed vector $\boldsymbol{a}_{d, 1}$ 's expectation is $\overline{\boldsymbol{a}_{d, 1}}=\left[\hat{\boldsymbol{h}_{I, d}}-\hat{\boldsymbol{h}_{R, d}} \tan \theta, \hat{\boldsymbol{h}_{R, d}}+\right.$ $\left.\hat{\boldsymbol{h}_{I, d}} \tan \theta\right]$, and its covariance matrix is given as $\boldsymbol{\Theta}_{d, 1}=$ $\operatorname{diag}(\underbrace{(1+\tan \theta)^{2} \sigma_{e}^{2}, \ldots,(1+\tan \theta)^{2} \sigma_{e}^{2}}_{2 N})$. Also, vector $\boldsymbol{a}_{d, 2}$ 's expectation is $\overline{\boldsymbol{a}_{d, 2}}=\left[-\boldsymbol{h}_{I, d}-\hat{\boldsymbol{h}_{R, d}} \tan \theta ;-\hat{\boldsymbol{h}_{R, d}}+\hat{\boldsymbol{h}_{I, d}} \tan \theta\right]$, with covariance matrix $\boldsymbol{\Theta}_{d, 2}=\boldsymbol{\Theta}_{d, 1}$. Hence, the two inequalities in (5) can be written as two cumulative probability functions in the form as $\Phi\left(\frac{-\sigma_{n} \sqrt{\overline{\Gamma_{d}}} \tan \theta-{\overline{\boldsymbol{a}_{d, 1}}}^{T}\left[\boldsymbol{u}_{R} ; \boldsymbol{u}_{I}\right]}{\left\|\Theta_{d, 1}^{\frac{1}{2}}\left[\boldsymbol{u}_{R} ; \boldsymbol{u}_{I}\right]\right\|_{2}}\right) \geq \eta_{d}$ and $\Phi\left(\frac{-\sigma_{n} \sqrt{\overline{\Gamma_{d}}} \tan \theta-{\overline{\boldsymbol{a}_{d, 2}}}^{T}\left[\boldsymbol{u}_{R} ; \boldsymbol{u}_{I}\right]}{\left\|\Theta_{d, 2}^{\frac{1}{2}}\left[\boldsymbol{u}_{R} ; \boldsymbol{u}_{I}\right]\right\|_{2}}\right) \geq \eta_{d}$, where $\Phi(\cdot)$ denotes the cumulative probability function of a standard normal distributed variable. Defining $\Phi^{-1}(\cdot)$ as the inverse function of $\Phi(\cdot),(5)$ can be written as

$$
\left\{\begin{array}{l}
{\overline{\boldsymbol{a}_{d, 1}}}^{T}\left[\boldsymbol{u}_{R} ; \boldsymbol{u}_{I}\right]+\Phi^{-1}\left(\eta_{d}\right)\left\|\Theta_{d, 1}^{\frac{1}{2}}\left[\boldsymbol{u}_{R} ; \boldsymbol{u}_{I}\right]\right\|_{2} \leq \varphi \\
{\overline{\boldsymbol{a}_{d, 2}}}^{T}\left[\boldsymbol{u}_{R}, \boldsymbol{u}_{I}\right]+\Phi^{-1}\left(\eta_{d}\right)\left\|\Theta_{d, 2}^{\frac{1}{2}}\left[\boldsymbol{u}_{R}, \boldsymbol{u}_{I}\right]\right\|_{2} \leq \varphi
\end{array}\right.
$$

where $\varphi=-\sigma_{n} \sqrt{\overline{\Gamma_{d}}} \tan \theta$. According to Schur Complements [17], Eq. (6) can be finally written as

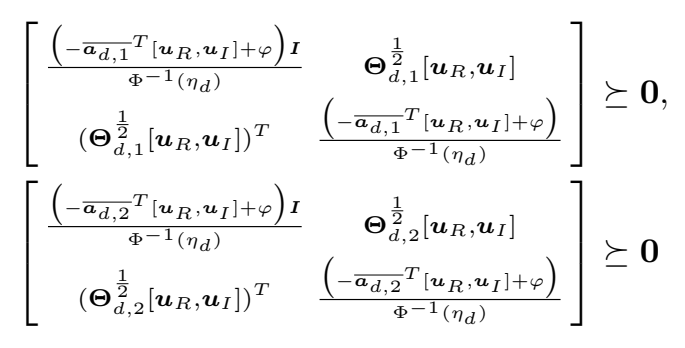

We now handle the security constraint against the $k$-th Eve in $(C 4)$ under the provision of destructive interference.
Confining the $k$-th Eve in the destructive region equals to $\left|\Im\left\{\boldsymbol{h}_{k}^{T} \boldsymbol{u}\right\}\right| \geq\left(\Re\left\{\boldsymbol{h}_{k}^{T} \boldsymbol{u}\right\}-\sigma_{n} \sqrt{\overline{\Gamma_{k}}}\right) \tan \theta$, and then the probabilistic constraint $(C 4)$ can be transformed into

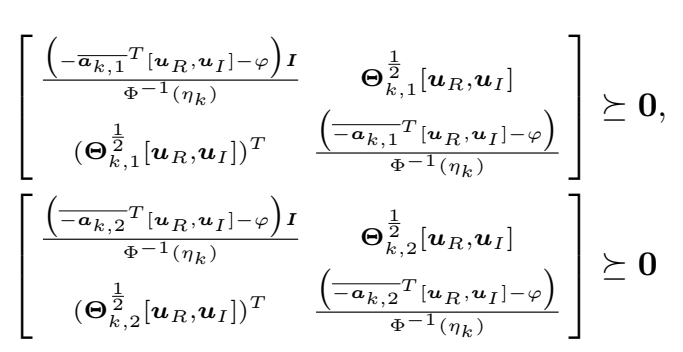

where $\overline{\boldsymbol{a}_{k, 1}}=\left[\hat{\boldsymbol{h}_{I, k}}+\hat{\boldsymbol{h}_{R, k}} \tan \theta, \hat{\boldsymbol{h}_{R, k}}-\hat{\boldsymbol{h}_{I, k}} \tan \theta\right]$ and $\overline{\boldsymbol{a}_{k, 2}}=$ $\left[-\hat{\boldsymbol{h}_{I, k}}+\hat{\boldsymbol{h}_{R, k}} \tan \theta,-\hat{\boldsymbol{h}_{R, k}}-\hat{\boldsymbol{h}_{I, k}} \tan \theta\right]$ with covariance ma$\operatorname{trix} \Theta_{k, 1}=\Theta_{k, 2}=\operatorname{diag}(\underbrace{(1+\tan \theta)^{2} \sigma_{e}^{2}, \ldots,(1+\tan \theta)^{2} \sigma_{e}^{2}}_{2 N})$.

(C3) and (C4) now have been handled into convex constraints. However, the optimization problem is still non-convex due to the binary variables in $(C 2): t_{n}=\{0,1\}, \forall n \in N$, which can be relaxed to the following two constraints [18]

$$
(C 2 a): t_{n}=[0,1], \forall n \in N,(C 2 b): \sum_{n=1}^{N} t_{n}-\sum_{n=1}^{N} t_{n}^{2} \leq 0,
$$

where $(C 2 a)$ is the relaxed version of the constraint $(C 2)$, and $(C 2 b)$ confines the value of $t_{n}$ close to 0 or 1 . Define $\boldsymbol{U}=\boldsymbol{u}^{H} \boldsymbol{u}$. Introducing a penalty factor $\varphi$, typically of large value, and moving $(C 2 b)$ into the objective function, the objective becomes into $\frac{\operatorname{Tr}(\boldsymbol{U})}{\alpha}+\sum_{n=1}^{N}\left(t_{n} p_{o n}+\left(1-t_{n}\right) p_{o f f}\right)+$ $\varphi\left(\sum_{n=1}^{N} t_{n}-\sum_{n=1}^{N} t_{n}^{2}\right)$, which shares the same optimal design policy and result with the original problem [18]. The last difficulty lies in the non-convex term $\varphi\left(\sum_{n=1}^{N} t_{n}-\sum_{n=1}^{N} t_{n}^{2}\right)$ in the objective term. It can be observed that $\sum_{n=1}^{N} t_{n}-$ $\sum_{n=1}^{N} t_{n}^{2}$ is the difference of two convex functions w.r.t the variable $t_{n}$, and thus can be handled by successive convex approximation such that $\sum_{n=1}^{N} t_{n}-\sum_{n=1}^{N} t_{n}^{2} \leq \sum_{n=1}^{N} t_{n}-$ $\sum_{n=1}^{N}\left(t_{n}^{(i)}\right)^{2}-2 \sum_{n=1}^{N} t_{n}^{(i)}\left(t_{n}-t_{n}^{(i)}\right)$, where $t_{n}^{(i)}$ denotes the value of $t_{n}$ at the $i$-th iteration [19]. Now, P1 becomes into

$$
\begin{aligned}
& P 2: \underset{\boldsymbol{u}, \boldsymbol{t}}{\operatorname{argmin}} \frac{\operatorname{Tr}(\boldsymbol{U})}{\alpha}+\sum_{n=1}^{N}\left(t_{n} p_{o n}+\left(1-t_{n}\right) p_{\text {off }}\right) \\
& \quad+\varphi\left(\sum_{n=1}^{N} t_{n}-\sum_{n=1}^{N}\left(t_{n}^{(i)}\right)^{2}-2 \sum_{n=1}^{N} t_{n}^{(i)}\left(t_{n}-t_{n}^{(i)}\right)\right), \\
& \text { s.t }(C 1),(C 2 a): t_{n}=[0,1], \forall n \in N,(C 3):(7), \\
& \quad(C 4):(8),(C 5):\left[\begin{array}{cc}
\boldsymbol{u} \\
\boldsymbol{u}^{T} & 1
\end{array}\right] \succeq 0,(C 6): \boldsymbol{U} \succeq 0 .
\end{aligned}
$$

Now P2 is a standard semi-definite programming problem, which can be solved by CVX [20]. To tighten the approximation, the value of $t_{n}^{(i)}$ is updated in an iterative way, i.e., we solve $\mathrm{P} 2$ and substitute the intermediate optimal value 
of $t_{n}^{*}$ to $t_{n}^{(i)}$ iteratively, $\forall n \in N$, until convergence. The successive convex approximation serves as the upper bound of the original problem, which is iteratively minimized and also lower bounded by the SINR and PHY security constraints. Hence, the convergence of the algorithm is confirmed.

\section{SIMULATION RESULTS}

We present the simulated performance in this section. The central frequency is set to $2 \mathrm{GHz}$ with $1 \mathrm{MHz}$ bandwidth. The AWGN power spectral density is $-174 \mathrm{dBm} / \mathrm{Hz}$. A $100 \times 100$ $\mathrm{m}^{2}$ square cell is considered with $N=16$ uniformly fixed DAs [4]. The IR and $K=14$ Eves are randomly distributed across the map. Drain efficiency of all DAs is set to $\alpha=40 \%$. Power consumption parameters are set to $p_{o n}=500 \mathrm{~mW}$, $p_{\text {off }}=50 \mathrm{~mW}$, and $p_{D A}=1000 \mathrm{~mW}$, respectively. CSI error is set to $\sigma_{d}=\sigma_{k}=0.01$. The PHY security against the Eves is set to $\overline{\Gamma_{k}}=-10 \mathrm{~dB}$, and probabilities are set to $\eta_{d}=\eta_{k}=0.95, \forall k \in K$. The PL model in [21] is adopted. Besides, the most related schemes, CA system without antenna selection (CA-no-AS) [11] and DA systems with conventional AN (DA-conv-AN) [2] are selected as benchmarks, while our proposed algorithm is named as DA-prob.

Fig. 2 a) shows the impact of the IR's SINR requirement $\overline{\Gamma_{d}}$ on the total power consumption. Firstly, it can be seen that the proposed algorithm outperforms the two benchmarks. It is because the proposed algorithm benefits from DAs' selection, the DAs far from the users may be deactivated for saving power. Hence, the proposed algorithm achieves a usercentric and on-demand network structure with higher degree of freedom over the CA-no-AS algorithm in [11]. Besides, our proposed algorithm rotates AN to make it constructive to the IR even with imperfect CSI, while AN is treated as an undesired element at the IR by conventional DA-conv-AN in [2]. Secondly, benefiting from geometric distribution of the DAs, the distances between the DAs and users are shortened and the system can always find near DAs to serve the users. With the alleviated PL as well as the lower required transmission power, DA can outperform its counterpart CA in terms of power efficient transmission. Thirdly, increasing the SINR threshold $\overline{\Gamma_{d}}$ leads to higher power consumption, as more antennas become activated and dissipate more circuit and transmission power. Also, the power consumption of the CA-noAS algorithm is dominated by its circuit power because of the fully activated antennas, and its power consumption increases slowly as the increased transmission power is overwhelmed by its circuit power consumption.

Fig. 2 b) shows the total power consumption with different number Eves. It can be seen that as the number of Eves increases, higher power of AN is needed to against the PHY security requirements $\overline{\Gamma_{k}}, \forall k \in K$, and thus total power consumption is increased as well. However, the total power consumption of the proposed algorithms increase slowly with more Eves, since AN contributes beneficially for the IR and
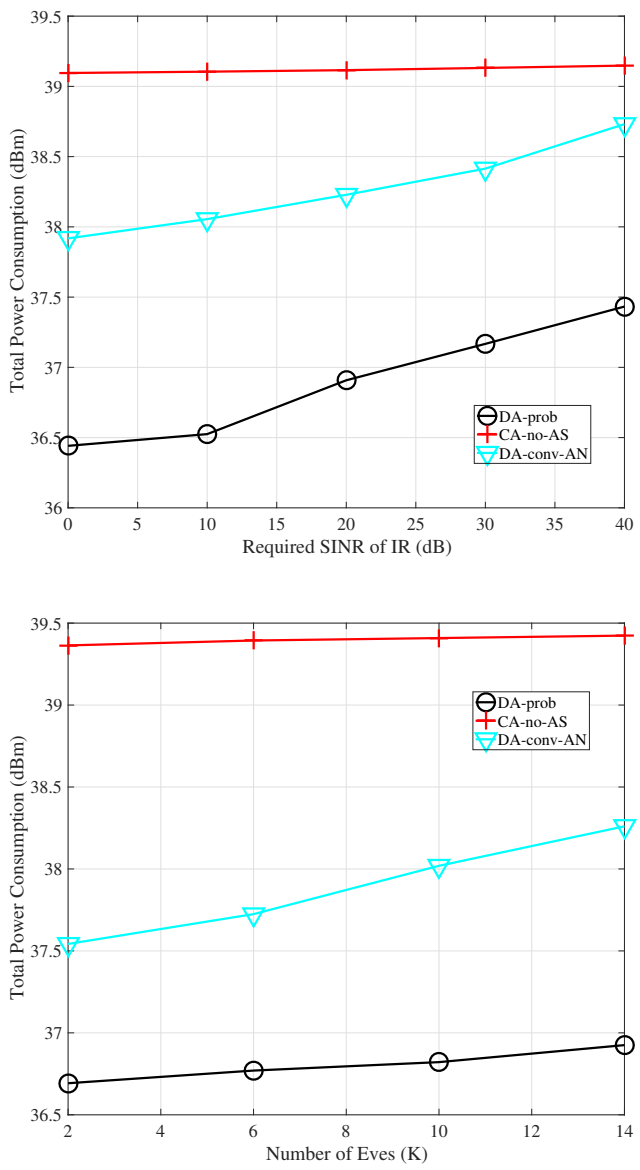

Fig. 2. a) The IR's SINR requirement vs. total power consumption. b) The number of Eves vs. total power consumption, where $\overline{\Gamma_{d}}=20 \mathrm{~dB}$.

the weight of precoder $\boldsymbol{w}$ may be relatively reduced to achieve the SINR target $\overline{\Gamma_{d}}$. By contrast, the two benchmarks consume higher power with more Eves, either hindered by the high circuit power consumption at the multiple antennas (CAno AS) or underutilized AN at the IR (DA-conv-AN).

\section{CONCLUSIONS}

To minimize total power consumption for DA systems with CSI uncertainties, antenna selection and precoding have been jointly optimized by probabilistic robust optimization. By the chance-constrained formulation, the proposed algorithm is able to satisfy the IR's QoS and simultaneously address PHY security against the Eves. Simulation has showed that the proposed algorithm consumes much lower power compared to the CA-no-AS and DA-conv-AN benchmarks. Furthermore, benefiting from the antenna selection mechanism in DA systems, a flexible and user-centric network structure is featured over its counterpart CA systems. 


\section{REFERENCES}

[1] H. Lei, M. Xu, I. S. Ansari, G. Pan, K. A. Qaraqe, and M. S. Alouini, "On secure underlay mimo cognitive radio networks with energy harvesting and transmit antenna selection," IEEE Trans. Green Commun. Netw., vol. 1, no. 2, pp. 192-203, Jun. 2017.

[2] D. W. K. Ng and R. Schober, "Secure and green swipt in distributed antenna networks with limited backhaul capacity," IEEE Trans. Wireless Commun., vol. 14, no. 9, pp. 5082-5096, Dec. 2015.

[3] Dilip Bethanabhotla, O. Y. Bursalioglu, H. C. Papadopoulos, and G. Caire, "Optimal user-cell association for massive mimo wireless networks," IEEE Trans. Wireless Commun., vol. 15, no. 3, pp. 1835-1850, Nov. 2015.

[4] Z. Wei, S. Sun, X. Zhu, Y. Huang, and J. Wang, "Energy efficient hybrid duplexing strategy for bidirectional distributed antenna systems," IEEE Trans. Veh. Technol., vol. 67, no. 6, pp. 5096-5110, Apr. 2018.

[5] Y. Huang, M. Liu, and Y. Liu, "Energy efficient swipt in iot distributed antenna systems," IEEE Internet of Thing Journal, vol. 5, no. 4, pp. 2646-2656, Aug. 2018.

[6] H. Wang, C. Wang, D. W. K. Ng, and M. H. Lee, "Artificial noise assisted secure transmission for distributed antenna systems," IEEE Trans. Signal Process., vol. 64, no. 15, pp. 4050-4061, Aug. 2016.

[7] S. Goel and R. Negi, "Guaranteeing secrecy using artificial noise," IEEE Trans. Wireless Common., vol. 7, no. 4, pp. 2180-2189, Jan. 2008.

[8] Q. Li and W. K. Ma, "Spatially selective artificial noise aided transmit optimization for miso multi-eves secrecy rate maximization," IEEE Trans. Signal Process., vol. 61, no. 10, pp. 2704-2717, May 2013.

[9] B. Zheng, M. Wen, C. Wang, X. Wang, F. Chen, J. Tang, and F. Ji, "Secure noma based two-way relay networks using artificial noise and full duplex," to appear in IEEE Journal on Sel. Area in Commun., vol. -, no. -, pp. 1-1, Dec. 2018.

[10] C. Masouros, "Correlation rotation linear precoding for mimo broadcast communications," IEEE Trans. Signal Process., vol. 59, no. 1, pp. 252-262, Jan. 2011.

[11] M. R. A. Khandaker, C. Masouros, and K. K. Wong, "Constructive interference based secure precoding: a new dimension in physical layer security," IEEE Trans. Inf. Foren. Sec., vol. 13, no. 9, pp. 2256-2268, Sept. 2018.
[12] K. Lee, J. P. Hong, H. H. Choi, and T. Q. S. Quek, "Secure noma based two-way relay networks using artificial noise and full duplex," IEEE Trans. Inf. Fore. and Sec., vol. 12, no. 1, pp. 162-174, Jan. 2019.

[13] Y. Sun, D. W. K. Ng, J. Zhu, and R. Schober, "Multiobjective optimization for robust power efficient and secure full-duplex wireless communication systems," IEEE Trans. Wireless Commun., vol. 15, no. 8, pp. 5515-5526, Aug. 2016.

[14] D. W. K. Ng and R. Schober, "Cross layer scheduling for ofdma amplify-and-forward relay network," IEEE Trans. Veh. Technol., vol. 59, no. 3, pp. 1443-1453, Mar. 2010.

[15] C. Masouros, T. Ratharajah, Mathini Sellathurai, C. B. Papadias, and A. K. S, "Known interference in the cellular downlink: a performance limiting or a source of green signal power,' IEEE Commun. Mag., vol. 51, no. 10, pp. 162-171, Oct. 2013.

[16] P. V. Amadori and C. Masouros, “Constant envelope precoding by interference exploitation in phase shift keying-modulated multiuser transmission," IEEE Trans. Wireless Commun., vol. 6, no. 1, pp. 538-550, Jan. 2017.

[17] S. Boyd and L. Vandenberghe, Convex Optimization, Cambridge University Press, Cambridge, U.K, 2 edition, 2005.

[18] D. W. K. Ng, Y. Wu, and R. Schober, "Power efficient resource allocation for full-duplex radio distributed antenna networks," IEEE Transactions on Wireless Commun., vol. 15, no. 4, pp. 2896-2911, Apr. 2016.

[19] D. Nguyen, L. N. Tran, P. Pirinen, and M. L. Aho, "Power efficient resource allocation for full-duplex radio distributed antenna networks," IEEE Transactions on Signal Process., vol. 61, no. 16, pp. 4038-4050, Aug. 2013.

[20] Z. Wei, X. Zhu, S. Sun, and J. Wang, "Energy efficient full duplex cooperative non-orthogonal multiple access," IEEE Trans. Veh. Technol., vol. 67, no. 10, pp. 10123-10128, Aug. 2018.

[21] J. Pardo, J. Rodrguez, and L. Llcer, "Polarized indoor mimo channel measurement at $2.45 \mathrm{ghz}$," IEEE Trans. Antenna Propagat., vol. 56, no. 12, pp. 3818-3828, Dec. 2006. 